\title{
COVQ for MAP Hard-Decision Demodulated Channels
}

\author{
Hamidreza Ebrahimzadeh Saffar, Student Member, IEEE, Fady Alajaji, Senior Member, IEEE, \\ and Tamás Linder, Senior Member, IEEE
}

\begin{abstract}
We design a channel optimized vector quantizer (COVQ) for symbol-by-symbol maximum a posteriori (MAP) hard-decision demodulated channels. The main objective is to exploit the non-uniformity of the indices representing the quantized source via the MAP decoder and iteratively optimize the overall discrete channel (at the symbol level) jointly with the quantizer. We consider memoryless Gaussian and Gauss-Markov sources transmitted over a binary phase-shift keying modulated Rayleigh fading channel. Our scheme has less encoding computational and storage complexity (particularly for noisy channel conditions) than both conventional and soft-decision COVQ systems, which use hard-decision and soft-decision maximum likelihood demodulation, respectively. Furthermore, it provides a notable signal-to-distortion ratio gain over the former system, and in some cases it matches or outperforms the latter one.
\end{abstract}

Index Terms-Joint source-channel coding, channel optimized vector quantization, MAP decoding, encoding computational and storage complexity, hard and soft-decision demodulation.

\section{INTRODUCTION}

$\mathbf{J}$ OINT source-channel coding (JSCC) has recently attracted considerable attention as it aims to surmount the limitations of classical tandem source-coding systems that are based on Shannon's separation theorem. Channel optimized vector quantization (COVQ) is a JSCC technique in which an analog source is quantized by taking into consideration the characteristics of both the source and the channel. COVQ has been thoroughly studied under different approaches (e.g., see [1] - [6] and [8] - [10]).

COVQ designs usually employ a discrete memoryless channel (DMC) corresponding to a memoryless continuous-valued channel used in conjunction with hard-decision demodulation. However, in these designs, little attention has been paid to optimize the discrete channel by exploiting the non-uniformity of the source encoder indices arriving at the channel input. A notable exception is [10] where non-iterative (one step) harddecision maximum a posteriori (MAP) decoding is considered.

In this work, we examine how to improve the performance of COVQ systems for hard-decision demodulated channels while decreasing the encoding computational and storage complexity. This is beneficial for wireless uplink communication applications (including sensor networks) where the scant resources of the transmitter need to be efficiently utilized. The conventional COVQ [5] and the soft-decision demodulation (SDD) COVQ [1], [2] and [6] (in which the channel soft-decision information is used) are based on maximum

Manuscript received September 11, 2008. The associate editor coordinating the review of this letter and approving it for publication was V. Stankovic. This work was supported in part by NSERC of Canada. All authors are with the Department of Mathematics and Statistics, Queen's University, Kingston, ON K7L 3N6, Canada (e-mail: \{hamid, fady, linder\}@mast.queensu.ca).

Digital Object Identifier 10.1109/LCOMM.2009.081457 likelihood (ML) decoding and have relatively low decoding computational complexity (although SDD COVQ can have considerably high storage requirements at the decoder). In this work, we relax the constraint on decoding complexity since we are interested in scenarios where, unlike the transmitter (e.g., a low-powered wireless sensor node), the receiver (e.g., the base station) has more than sufficient processing power. We will focus on binary phase shift keying (BPSK) modulated Rayleigh fading channels, although the algorithm can also be applied to other channels [3]. We iteratively optimize the model DMC (having identical input and output alphabets) representing the concatenation of the modulator, channel and hard-decision demodulator together with its correspondingly designed COVQ encoder/decoder pair. This is achieved by using a symbol-bysymbol MAP hard-decision detector instead of the standard maximum likelihood (ML) detector, motivated by the fact that the COVQ encoder indices arriving at the modulator are non-uniformly distributed (hence MAP decoding outperforms ML decoding as it minimizes the discrete channel's symbol error rate). We propose an iterative three-phase COVQ design algorithm which is based on first designing a conventional COVQ, then computing the input (quantizer index) distribution for the use in MAP decoding, and finally redesigning the COVQ for the new channel defined in terms of the updated transition matrix.

Numerical results indicate notable signal-to-distortion ratio (SDR) and encoding complexity gains for the proposed scheme over the conventional and SDD COVQ schemes.

\section{COVQ SYSTEM}

The general block diagram of the system is depicted in Fig. 1. The purpose of the system is to transmit the random vector $\boldsymbol{X}_{n} \in \mathbb{R}^{k}$ of dimension $k$ over the noisy channel and form an estimate $\hat{\boldsymbol{X}}_{n}$ of $\boldsymbol{X}_{n}$ based on the channel output $\boldsymbol{R}_{n}$, such that the distortion $\mathbb{E}\left\|\boldsymbol{X}_{n}-\hat{\boldsymbol{X}}_{n}\right\|^{2}$ is minimized. Here, $n$ represents the time index of the vector which consists of $k$ single source outputs. The source $\left\{\boldsymbol{X}_{n}\right\} \in \mathbb{R}^{k}$ is assumed to be a stationary and ergodic process, with zero mean and unit variance. The COVQ encoder encodes $\left\{\boldsymbol{X}_{n}\right\}$ at a rate of $r$ bits per sample (bps). Therefore, the COVQ encoder is a mapping $\mathscr{E}: \mathbb{R}^{k} \rightarrow \mathcal{I}_{n} \triangleq\left\{0,1, \cdots, N_{e}-1\right\}$, such that $\mathscr{E}\left(\boldsymbol{X}_{n}\right)=I_{n}$ is sent over the channel after modulation $\left(N_{e}=2^{k r}\right)$. The encoding is done using the partition $\mathcal{P}=\left\{\mathcal{S}_{i}\right\}_{i=0}^{N_{e}-1}$ of $\mathbb{R}^{k}$ via the encoding rule: $\boldsymbol{X}_{n} \in \mathcal{S}_{i} \Rightarrow I_{n}=\mathscr{E}\left(\boldsymbol{X}_{n}\right)=i$. Note that for simplicity, we also identify the index $I_{n}$ and the index set $\mathcal{I}_{n}$ with their binary representations (e.g., $\mathcal{I}_{n}=\{0,1\}^{k r}$ ). The input index probability distribution is denoted by $P_{i}$ for $i=$ $0,1, \cdots, N_{e}-1$. We use BPSK modulation, although other memoryless modulation techniques can also be considered. 
Equivalent Discrete Memoryless Channel (DMC)

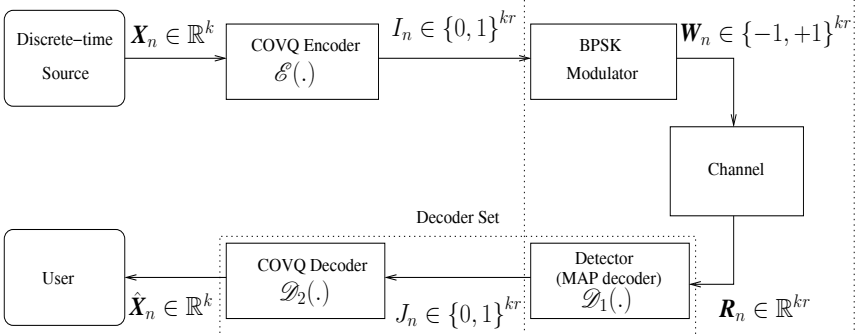

Fig. 1. Block diagram of the iterative MAP decoded COVQ system.

The concatenation of the modulator, the actual channel and the detector (at the symbol level) forms a DMC, for which the COVQ is designed. We refer to this discrete channel as the "equivalent DMC." The DMC is defined in terms of its input alphabet $\mathcal{I}_{n}=\left\{0,1, \cdots, N_{e}-1\right\}$, transition matrix $P_{Y \mid X}$ and the output alphabet $\mathcal{J}_{n}=\left\{0,1, \cdots, N_{d}-1\right\}$. Since the system uses hard-decision demodulation, $\mathcal{J}_{n}=\mathcal{I}_{n}$ and $N_{d}=N_{e}=N$. (In SDD COVQs ( [1], [2] and [6]), one has $N_{d}=N_{e}^{q}>N_{e}$. This is achieved by a soft-decision $2^{q}$-level uniform scalar quantizer at the decoder).

The decoder is the composition of two functions $\mathscr{D}_{1}$ and $\mathscr{D}_{2}$. Thus the decoder can be written as $\mathscr{D}=\mathscr{D}_{2} \circ \mathscr{D}_{1}$, where $\mathscr{D}_{1}: \mathbb{R}^{k r} \rightarrow \mathcal{J}_{n}=\mathcal{I}_{n}=\left\{0,1, \cdots, N_{e}-1\right\}, \mathscr{D}_{2}: \mathcal{J}_{n} \rightarrow$ $\mathcal{C} \subset \mathbb{R}^{k}$, o denotes function composition and $\mathcal{C}$ is called the codebook and its entries are called codevectors.

The BPSK modulated bits with unit energy to be sent over the Rayleigh fading channel are denoted by $W_{n}^{1}, W_{n}^{2}, \cdots, W_{n}^{k r}$ and form the vector $\boldsymbol{W}_{n} \in\{-1,+1\}^{k r}$. At the output of the channel, the received vector $\boldsymbol{R}_{n}$ consists of $k r$ components $R_{n}^{1}, R_{n}^{2}, \cdots, R_{n}^{k r}$ that can each be written as $R_{n}^{t}=h_{t} W_{n}^{t}+\nu_{t}$, for $t=1,2, \cdots, k r$, where $\left\{\nu_{t}\right\}$ is the independent and identically distributed (i.i.d) Gaussian noise: $\nu_{t} \sim \mathcal{N}\left(0, \frac{N_{0}}{2}\right)$, and $\left\{h_{t}\right\}$ is the i.i.d Rayleigh distributed fading process with $\mathbb{E}\left[h_{t}^{2}\right]=1$. We also assume that the processes $h_{t}, \nu_{t}$ and $W_{n}^{t}$ are independent of each other and that the fading amplitude values $h_{t}$ are perfectly known at the receiver (i.e., we assume perfect channel side information at the decoder side).

\section{Three-Phase Iterative MAP Decoded (IMD) ALGORITHM}

The main contribution of this paper is a simple algorithm that jointly optimizes $\mathscr{D}_{1}$ and the pair $\left\{\mathscr{D}_{2}, \mathscr{E}\right\}$. The IMD algorithm consists of three phases as follows.

First phase: The first phase is the ordinary COVQ design algorithm. The problem of designing COVQ for a DMC is well known (e.g., see [4], [5] and [6]). Starting from a suitable initial codebook (decoder) the COVQ encoder and decoder are iteratively and alternatingly optimized based on two necessary conditions [5] for minimizing the squared-error distortion, making sure that the procedure ends up with a locally optimal solution. Thus, in the first phase, we use the COVQ design algorithm [5] where $\mathscr{D}_{1}$ is fixed and $\mathscr{E}$ and $\mathscr{D}_{2}$ are optimized in an iterative fashion.

Note that in the first step of the iteration, we assume a uniform input index distribution resulting in ML decoding. In this case, the DMC is a binary symmetric channel (BSC) used $k r$ times.

Second phase: Once Phase 1 is complete, the encoder index distribution is fed to the MAP decoder to start the second phase of the algorithm. We use the computed input distribution to replace the ML detector by a symbol based MAP decoder and redesign the COVQ. Given $\mathscr{D}_{2}$ and $\mathscr{E}$ from the first phase, we find $\mathscr{D}_{1}$ such that

$$
\begin{aligned}
J_{n} & =\arg \max _{I_{n} \in \mathcal{I}_{n}} P\left(I_{n} \mid \boldsymbol{R}_{n}, \mathbf{h}\right)=\arg \max _{\mathcal{I}_{n}} P\left(\boldsymbol{R}_{n} \mid I_{n}, \mathbf{h}\right) P_{I_{n}} \\
& =\arg \max _{I_{n} \in \mathcal{I}_{n}} P\left(\left\{\boldsymbol{\nu}=\boldsymbol{R}_{n}-\mathbf{h} \odot \boldsymbol{W}_{n}\left(I_{n}\right)\right\} \mid I_{n}, \mathbf{h}\right) P_{I_{n}} \\
& =\arg \min _{I_{n} \in \mathcal{I}_{n}}\left[\frac{1}{N_{0}}\left\|\boldsymbol{R}_{n}-\mathbf{h} \odot \boldsymbol{W}_{n}\right\|^{2}-\ln P_{I_{n}}\right], \quad \text { (1) }
\end{aligned}
$$

where $\boldsymbol{W}_{n}\left(I_{n}\right)$ denotes the symbol corresponding to index $I_{n}$, $\mathbf{h}$ and $\boldsymbol{\nu}$ denote the fading and noise vectors $\left(h_{1}, h_{2}, \cdots, h_{k r}\right)$ and $\left(\nu_{1}, \nu_{2}, \cdots, \nu_{k r}\right)$, respectively, and $\odot$ represents elementwise vector multiplication.

Based on the above MAP metric, the new transition matrix of the equivalent DMC is empirically computed. It can be seen that the transition matrix $P_{J_{n} \mid I_{n}}$ is a function of $\mathbf{h}$ and is thus time-variant. Using these transition matrices would result in separate decoder for each value of $\mathbf{h}$, a clearly unfeasible solution. Hence to curb the complexity of the system, for design purposes, we compute and use the average of the channel transition distribution matrices over several fading vectors (i.e., we compute $\mathbb{E}_{\mathbf{h}}\left(P_{J_{n} \mid I_{n}}\right)$ ).

After updating the detector, which results in a new DMC, and finding an updated codebook (based on the new DMC probability distribution), we design the new COVQ (the pair $\left\{\mathscr{D}_{2}, \mathscr{E}\right\}$ ), using the updated codebook found previously as the initial codebook of the GLA. We calculate the distortion $D_{n}$ at the end of the second phase.

Third phase: In the third phase, we iterate Phase 2 and terminate when the SDR is maximized.

\section{ENCODER COMPLEXITY}

An important advantage of the IMD COVQ is its reduced encoding computational and storage complexity when compared with other schemes. We measure the computational complexity by the number of multiplications required to encode one source sample (as in [7]), while the storage requirement is measured by the total number of scalars needed to be stored at the encoder [7].

It is important to note that the encoding computational complexity of COVQ systems changes with the channel signalto-noise ratio (SNR). It is well known (e.g., [5] and [3]) that in COVQ systems, the number of non-empty encoding cells ${ }^{1}$ considerably decreases for very low SNRs and the computational complexity is proportional to the number of non-empty regions, which we denote by $N_{\bar{\varnothing}}$. From the form of the encoder function $\mathscr{E}$, it can be shown [3] that the computational complexity for each of the COVQ, IMD COVQ and SDD COVQ systems equals $N_{\bar{\varnothing}}$. It can also be shown that

\footnotetext{
${ }^{1}$ An empty cell (as opposed to a non-empty cell) refers to a corresponding encoding region of an index for which for every input training vector there is at least one other index with smaller encoding function metric. This index is not sent at all and its corresponding region is empty.
} 
TABLE I

SDR AND THE NUMBER OF NON-EMPTY ENCODING CELLS (ENCODING COMPLEXITY) FOR THE MEMORYLESS GAUSSIAN $(\rho=0.0)$ AND GAUSS-MARKOV ( $\rho=0.9)$ SOURCES AND DIFFERENT COVQ SYSTEMS. THE ENCODER RATE IS $r=2$ BPS AND THE QUANTIZATION DIMENSION IS $k=2$.

\begin{tabular}{|c|c|c|c|c|c|c|c|c|c|c|c|c|}
\hline \multirow{3}{*}{\begin{tabular}{|l} 
Channel \\
SNR \\
$(\mathrm{dB})$
\end{tabular}} & \multicolumn{6}{|c|}{$\mathrm{SDR}(\mathrm{dB})$} & \multicolumn{6}{|c|}{ Number of Non-Empty Cells $\left(N_{\bar{\varnothing}}\right)$} \\
\hline & \multicolumn{2}{|c|}{ COVQ } & \multicolumn{2}{|c|}{$\begin{array}{c}\text { IMD } \\
\text { COVQ }\end{array}$} & \multicolumn{2}{|c|}{$\begin{array}{c}\text { SDD COVQ } \\
(q=2)\end{array}$} & \multicolumn{2}{|c|}{ COVQ } & \multicolumn{2}{|c|}{$\begin{array}{c}\text { IMD } \\
\text { COVQ }\end{array}$} & \multicolumn{2}{|c|}{$\begin{array}{c}\text { SDD COVQ } \\
(q=2)\end{array}$} \\
\hline & $\rho=0.0$ & $\rho=0.9$ & $\rho=0.0$ & $\rho=0.9$ & $\rho=0.0$ & $\rho=0.9$ & $\rho=0.0$ & $\rho=0.9$ & $\rho=0.0$ & $\rho=0.9$ & $\rho=0.0$ & $\rho=0.9$ \\
\hline 8.0 & 4.93 & 6.60 & 5.28 & 7.46 & 5.63 & 7.31 & 16 & 11 & 13 & 8 & 16 & 11 \\
\hline 6.0 & 4.08 & 5.55 & 4.43 & 6.53 & 4.75 & 6.30 & 16 & 10 & 13 & 8 & 16 & 10 \\
\hline 4.0 & 3.23 & 4.45 & 3.66 & 5.43 & 3.84 & 5.43 & 16 & 9 & 11 & 7 & 14 & 9 \\
\hline 3.0 & 2.83 & 3.92 & 3.23 & 4.84 & 3.41 & 4.85 & 16 & 9 & 11 & 7 & 14 & 9 \\
\hline 2.0 & 2.46 & 3.43 & 2.85 & 4.31 & 3.00 & 4.28 & 16 & 8 & 11 & 7 & 14 & 8 \\
\hline 1.0 & 2.13 & 2.94 & 2.49 & 3.67 & 2.74 & 3.72 & 16 & 8 & 11 & 7 & 14 & 8 \\
\hline 0.0 & 1.85 & 2.70 & 2.11 & 3.42 & 2.30 & 3.21 & 16 & 7 & 11 & 6 & 14 & 7 \\
\hline-1.0 & 1.57 & 2.33 & 1.75 & 2.88 & 1.97 & 2.73 & 16 & 7 & 11 & 6 & 14 & 7 \\
\hline-2.0 & 1.32 & 1.93 & 1.49 & 2.54 & 1.68 & 2.31 & 15 & 6 & 11 & 5 & 14 & 6 \\
\hline-3.0 & 1.10 & 1.77 & 1.21 & 2.14 & 1.41 & 1.94 & 14 & 6 & 11 & 5 & 14 & 6 \\
\hline-4.0 & 0.90 & 1.51 & 1.01 & 1.83 & 1.23 & 1.77 & 14 & 6 & 11 & 5 & 14 & 5 \\
\hline-6.0 & 0.61 & 0.99 & 0.69 & 1.23 & 0.79 & 1.22 & 12 & 5 & 9 & 5 & 14 & 5 \\
\hline
\end{tabular}

the encoding storage requirement for each of the COVQ, IMD COVQ and SDD COVQ systems is given by $(k+1) N_{\bar{\varnothing}}$ [3]. Thus we use the parameter $N_{\bar{\varnothing}}$ as a criterion to compare the encoding computational and storage efficiency of the systems.

\section{Numerical Results}

In the first phase (COVQ for hard-decision ML decoded channel), we employ the transition matrix derived from $\mathrm{kr}$ uses of a BSC with crossover probability

$$
\mathbb{E}_{h}\left[P_{J_{n} \mid I_{n}}(1 \mid 0)\right]=\mathbb{E}_{h}\left[P\left(\nu_{t}>h_{t}\right)\right]=\mathbb{E}_{h}[Q(h \sqrt{\mathrm{SNR}})],
$$

where SNR $=\mathbb{E}\left[W_{t}^{2}\right] / \mathbb{E}\left[\nu_{t}^{2}\right]=\frac{2}{N_{0}}$ and $Q(\cdot)$ is the complementary error function $Q(x) \triangleq(1 / \sqrt{2 \pi}) \int_{x}^{\infty} \exp \left\{-\tau^{2} / 2\right\} d \tau$. For designing the COVQ, 100,000 source training vectors are generated. After designing the conventional COVQ (Phase 1), we generate 400,000 noise vectors, use MAP decoding and from the resulting empirical distribution compute the new $2^{k r} \times 2^{k r}$ DMC transition matrix. The matrix is calculated for 2000 fading vectors $\mathbf{h}$ and the arithmetic average of all matrices is used as the updated transition matrix. Then the COVQ is redesigned as described in Phases 2 and 3 above.

We compare the performance and encoder complexity of the conventional COVQ with the proposed IMD COVQ and the 4-level $(q=2)$ SDD COVQ [1]. Table I presents SDR and $N_{\bar{\varnothing}}$ results for a memoryless Gaussian (with correlation coefficient $\rho=0.0$ ) source and highly correlated Gauss-Markov source ( $\rho=0.9$ ) for quantization dimension $k=2$ and rate $r=2$ bps. Table II presents SDR and $N_{\bar{\varnothing}}$ results for a memoryless Gaussian source with a higher dimension of $k=3$ and rate $r=2$ bps. It can be seen from the tables that there is a considerable SDR gain for IMD COVQ over conventional COVQ, while it sometimes matches or outperforms the SDD COVQ (up to $1.79 \mathrm{~dB}$ gain over COVQ and up to 0.26 $\mathrm{dB}$ gain over SDD COVQ for SNR $=4 \mathrm{~dB}$ as shown in Table II). This gain increases for higher correlation coefficients and higher values of $k r$. Interestingly, we observe that in general, after applying the IMD algorithm, the non-uniform input distribution, tends to be even more non-uniform which
TABLE II

SDR AND THE NUMBER OF NON-EMPTY ENCODING CELLS (ENCODING COMPLEXITY) FOR THE MEMORYLESS GAUSSIAN SOURCE $(\rho=0.0)$ AND DIFFERENT COVQ SYSTEMS. THE ENCODER RATE IS $r=2$ BPS AND THE QUANTIZATION DIMENSION IS $k=3$.

\begin{tabular}{||c|c|c|c|c|c|c|c||}
\hline \hline \multicolumn{2}{|c|}{ Channel SNR (dB) } & -4 & -2 & 0 & 2 & 4 & 6 \\
\hline \multirow{3}{*}{ SDR $(\mathrm{dB})$} & COVQ & 0.94 & 1.40 & 1.93 & 2.60 & 3.36 & 4.22 \\
\cline { 2 - 8 } & IMD COVQ & 1.23 & 1.86 & 2.97 & 3.88 & 5.15 & 5.69 \\
\cline { 2 - 8 } & SDD COVQ $(q=2)$ & 1.32 & 1.94 & 2.88 & 3.79 & 4.89 & 5.78 \\
\hline Number & COVQ & 54 & 52 & 55 & 60 & 64 & 64 \\
\cline { 2 - 8 } of Non-Empty & IMD COVQ & 31 & 34 & 26 & 45 & 54 & 58 \\
\cline { 2 - 8 } Cells $\left(N_{\bar{\varnothing}}\right)$ & SDD COVQ $(q=2)$ & 52 & 49 & 58 & 59 & 64 & 64 \\
\hline \hline
\end{tabular}

is desirable [5], thus improving the IMD COVQ system over the conventional COVQ and SDD COVQ systems in terms of encoding computational complexity $\left(N_{\bar{\varnothing}}\right)$ and encoding storage requirements $\left((k+1) N_{\bar{\varnothing}}\right)$. In terms of the number $N_{\bar{\varnothing}}$ of non-empty cells, IMD COVQ outperforms both the conventional COVQ and the SDD COVQ considerably (up to $53 \%$ reduction over conventional COVQ and up to $55 \%$ reduction over SDD COVQ for SNR $=0 \mathrm{~dB}$ as shown in Table II). Since there are already many empty decision regions for the conventional COVQ at very low SNRs (less than $-2 \mathrm{~dB}$ ), the IMD algorithm does not provide much gain (either in performance or encoder complexity) in that region. However, for SNR ranging from $-2 \mathrm{~dB}$ to $4 \mathrm{~dB}$, it gives maximum gain over the other schemes. For high SNRs, MAP decoding does not yield much gain compared with ML decoding as in this case these two decoding methods are nearly equivalent. Finally, it is worth pointing out that while we cannot analytically guarantee the convergence of the proposed algorithm, we did observe a rapid convergence in our experiments, with the final solution reached in only a few (up to 4) steps [3].

\section{REFERENCES}

[1] F. Alajaji and N. Phamdo, "Soft-decision COVQ for Rayleigh-fading channels," IEEE Commun. Lett., vol. 2, pp. 162-164, June 1998.

[2] F. Behnamfar, F. Alajaji, and T. Linder, "Channel-optimized quantization with soft-decision demodulation for space-time orthogonal block-coded channels," IEEE Trans. Signal Processing, vol. 54, no. 10, pp. 39353946, Oct. 2006

[3] H. Ebrahimzadeh Saffar, "Channel optimized vector quantization: iterative design algorithms," Master's thesis, Queen's University, 2008.

[4] N. Farvardin, "A study of vector quantization for noisy channels," IEEE Trans. Inform. Theory, vol. 36, pp. 799-809, July 1990.

[5] N. Farvardin and V. Vaishampayan "On the performance and complexity of channel optimized vector quantizers," IEEE Trans. Inform. Theory, vol. 37, pp. 155-160, Jan. 1991.

[6] N. Phamdo and F. Alajaji, "Soft-decision demodulation design for COVQ over white, colored, and ISI Gaussian channels," IEEE Trans. Commun., vol. 48, no. 9, pp. 1499-1506, Sept. 2000.

[7] D. S. Kim and N. B. Shroff, "Quantization based on a novel sample adaptive product quantizer (SAPQ)," IEEE Trans. Inform. Theory, vol. 45, pp. 2306-2320, Nov. 1999.

[8] M. Skoglund, "Soft decoding for vector quantization over noisy channels with memory," IEEE Trans. Inform. Theory, vol. 45, pp. 12931307, May 1999.

[9] N. Wernersson and M. Skoglund, "On source decoding based on finitebandwidth soft information," in Proc. IEEE Int. Symp. Inform. Theory, Adelaide, Australia, Sept. 2005, pp. 87-91.

[10] P. Yahampath and M. Pawlak, "Vector quantization for finite-state Markov channels and application to wireless communications," Eur. Trans. Telecommun., vol. 18, no. 4, pp. 327-342, Feb. 2007. 